\title{
Dynamical Masses of Young Star Clusters: Constraints on the Stellar IMF and Star-Formation Efficiency
}

\author{
N. Bastian \\ Department of Physics and Astronomy, University College London, Gower Street, \\ London WC1E 6BT, UK \\ email: bastian@star.ucl.ac.uk
}

\begin{abstract}
Through the use of detailed light profiles and dynamical measurements of young clusters we investigate claims that the stellar initial mass function within clusters varies greatly. We find a strong age dependence in the clusters which have been claimed to have non-standard stellar IMFs, and suggest that the lack of equilibrium of these clusters is responsible for their 'strange' light-to-mass ratios and not IMF variations. The most likely culprit is the rapid removal of residual gas left over from the star-formation process which leaves the clusters severely out of dynamical equilibrium. By comparing the observations to N-body simulations we quantify to what degree a cluster is out of equilibrium and consequently its survival chances. We find that $>60 \%$ of young clusters will be disrupted, due gas removal, within the first 20-50 Myr of their lives.
\end{abstract}

Keywords. galaxies: star clusters

\section{The Stellar Initial Mass Function in Clusters}

Many recent works have attempted to constrain the stellar initial mass function (IMF) inside massive clusters by comparing their dynamical mass estimates (found through measuring the velocity dispersion and effective radius) to the measured light. These studies have come to different conclusions, with some claiming standard Kroupa-type (Kroupa 2002) IMFs (e.g. Maraston et al. 2004) while others have claimed extreme nonstandard IMFs (e.g. the top or bottom of the IMF is over-populated with respect to a Kroupa IMF (Smith \& Gallagher 2001). However, the results appear to be correlated with the age of the clusters, as older clusters (>80 Myr) all appear to be well fit by a Kroupa-type IMF whereas younger clusters display significant scatter in their best fitting IMF (Bastian et al. 2006). This has led to the suggestion that the younger clusters are out of Virial equilibrium, thus undercutting the fundamental assumption which is necessary to derive dynamical masses. We will return to this point in $\S 2$ and $\S 3$ Focusing on the older clusters, we see that they all have standard IMFs (see Fig. 2), arguing that at least in massive clusters the IMF does not vary significantly.

\section{Dynamical Equilibrium of Young Clusters}

One explanation of why the youngest clusters are not in dynamical equilibrium is that young clusters are expected to expel their remaining gas (left over from the starformation process) on extremely rapid timescales, which will leave the cluster severely out of equilibrium (e.g. Goodwin 1997). In order to search for such an effect we compared the luminosity profiles of three young clusters with that of N-body simulations of clusters which are undergoing violent relaxation due to rapid gas loss (Bastian \& Goodwin 2006). 


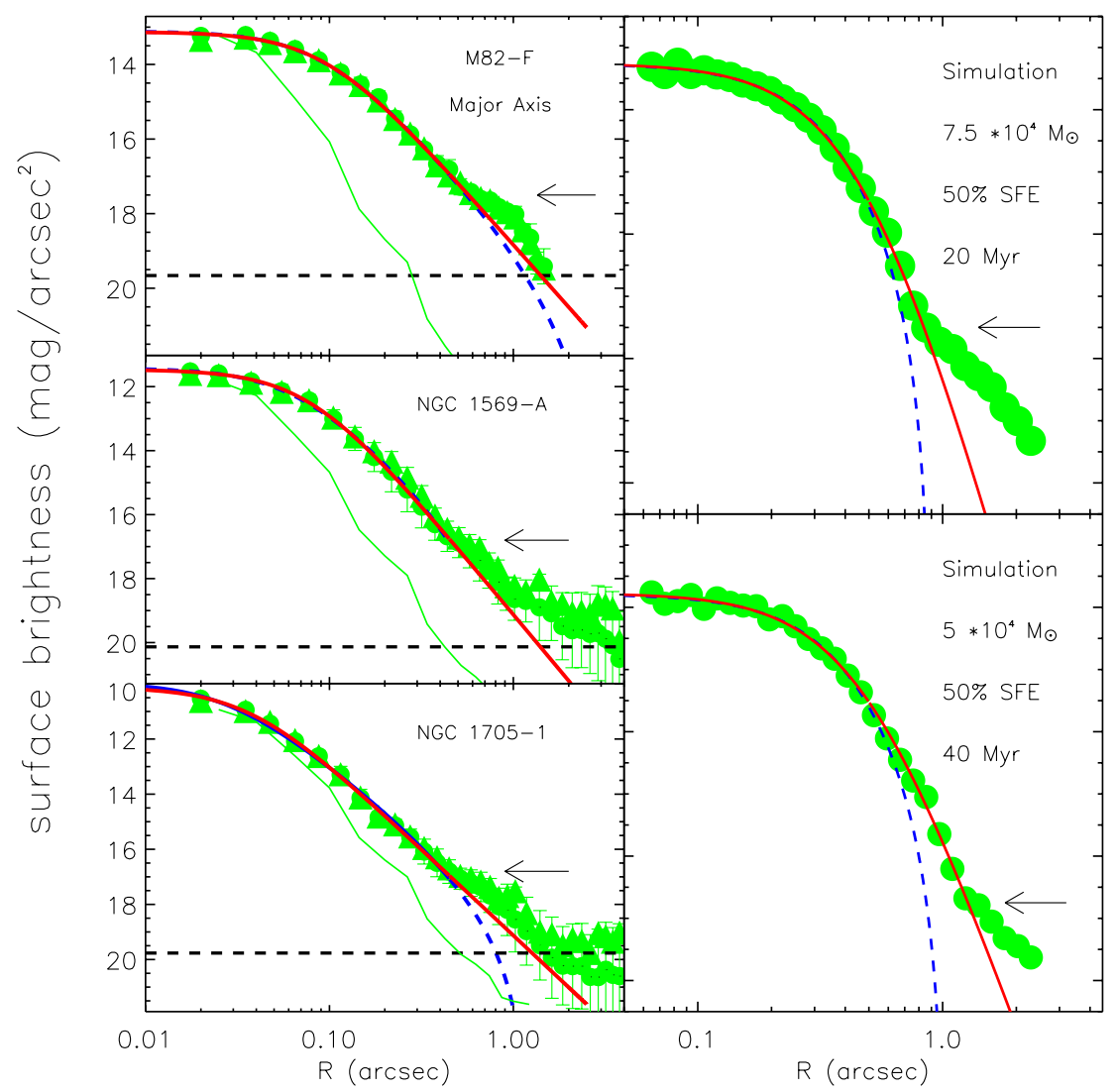

Figure 1. Taken from Bastian \& Goodwin (2006): Surface brightness profiles for three young clusters (left - M82-F, NGC 1569-A, and NGC 1705-1) and two N-body simulations which include the rapid removal of gas which was left over from a non-100\% star-formation efficiency (right). The solid (red) and dashed (blue) lines are the best fitting EFF (Elson, Fall, \& Freeman 1987) and King (1962) profiles respectively. Note the excess of light at large radii with respect to the best fitting EFF profile in both the observations and models. This excess light is due to an unbound expanding halo of stars caused by the rapid ejection of the remaining gas after the cluster forms. Hence, excess light at large radii strongly implies that these clusters are not in dynamical equilibrium. For details of the modelling and observations see Bastian \& Goodwin (2006) and Goodwin \& Bastian (2006).

The simulations (Fig. 1, right panel) make the generic prediction of excess light at large radii (with respect to the best fitting EFF profile (Elson, Fall, \& Freeman 1987), due to an unbound expanding halo of stars which stays associated with the cluster for $20-50$ Myr. These stars are unbound due to the rapid decrease of potential energy as the gas is removed on timescales shorter than a crossing time (e.g. Goodwin 1997). Observations of the three young clusters also show excess light at large radii (Fig. 1, left panel), strongly suggesting that they are experiencing violent relaxation (Bastian \& Goodwin 2006). Hence these clusters are not in dynamical equilibrium.

\section{The Star Formation Efficiency and Infant Mortality}

Assuming that young clusters are out of equilibrium due to rapid gas loss (the extent of which is determined by the star-formation efficiency - SFE one can fold these effects 


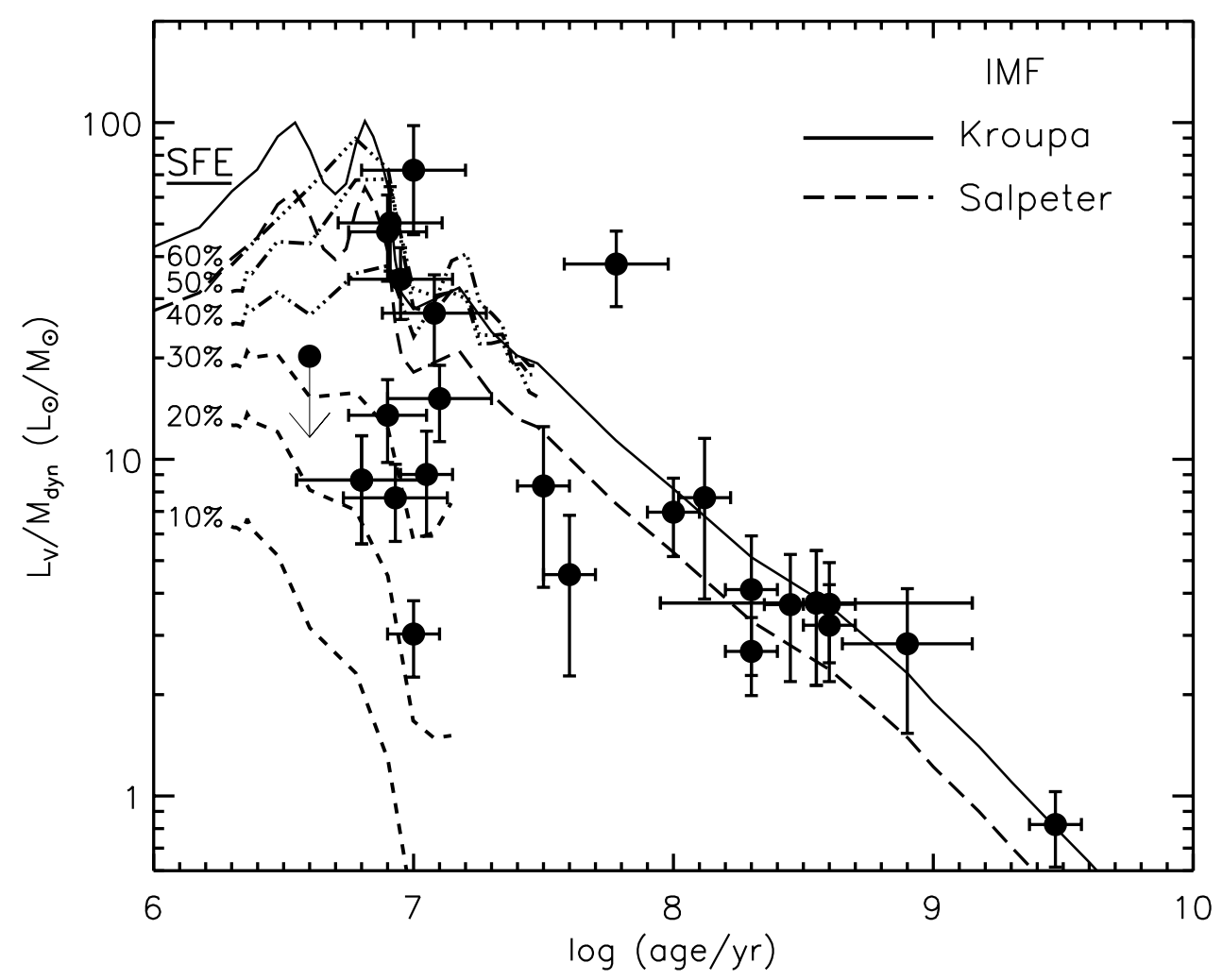

Figure 2. Taken from Goodwin \& Bastian (2006): The light-to-mass ratio of young clusters. The data are from Goodwin \& Bastian (2006) and references therein. The solid line is the prediction of simple stellar population models (SSPs) with a Kroupa (2002) stellar IMF wile the long-dashed line shows the same but for a Salpeter (1995) IMF. The short-dashed and dash-dotted lines are the SSP model tracks folded with the effects of rapid gas removal following non-100\% star-formation efficiencies (SFE). Short-dashed lines represent the SFEs where the clusters will become completely unbound. The SFE in the simulations measures the degree to which the cluster is out-of-virial equilibrium after gas loss, and so is an effective SFE (see Goodwin \& Bastian 2006).

(see Fig. 3 in Bastian \& Goodwin 2006) into SSP models (Goodwin \& Bastian 2006). The results are shown as solid and dashed red lines in Fig. 2 for various SFEs, where we have assumed all gas is lost instantaneously at $2 \mathrm{Myr}$. The dashed lines show the results for SFEs below 30\% for which the cluster will become completely unbound. Solid lines represent SFEs above $30 \%$ where a bound core may remain. Note that the observed SFEs of the clusters range from 10-60\% (Goodwin \& Bastian 2006).

We also note that 7 out of the 12 clusters with ages below $20 \mathrm{Myr}$ appear unbound (i.e. SFE $<30 \%$ ), suggesting that $\sim 60 \%$ of clusters will become unbound in the first 20-50 Myr of their lives Goodwin \& Bastian (2006), i.e. what has been termed "infant mortality". This is in close agreement with cluster population studies of M51 which found an infant mortality rate of $68 \%$ (Bastian et al. 2005) and comparable to the open cluster dispersal rate of $\sim 87 \%$ (Lada \& Lada 1991, see also Whitmore 2003 and Pellerin et al. 2007). 


\section{Conclusions}

Through detailed comparisons of the luminosity profiles of young clusters with N-body simulations of clusters including the effects of rapid gas loss, we argue that young clusters are not in Virial equilibrium. This undercuts the fundamental assumption needed to determine dynamical masses. This suggests that the claimed IMF variations are probably due to the internal dynamics of the clusters and not related to the IMF. By limiting the sample to the oldest clusters (which appear to be in equilibrium) we see that they are all well fit by a Kroupa-type IMF arguing that, at least in massive star clusters, the IMF does not vary significantly.

By combining the above N-body simulations with SSP models we can derive the (effective) SFE of clusters. From this we find that $\sim 60 \%$ of young clusters appear to be unbound, in good agreement with other estimates of the infant mortality rate. Note however that even if a cluster survives this phase it may not survive indefinitely due to internal and external effects (e.g. Gieles et al. 2005).

\section{Acknowledgements}

NB gratefully acknowledges Simon Goodwin for his large part in the studies presented here.

\section{References}

Bastian, N., Gieles, M., Lamers, H. J. G. L. M., Scheepmaker, R. A., \& de Grijs, R. 2005, AछA 431,905

Bastian, N. \& Goodwin, S. P. 2006, MNRAS, 369, L9

Bastian, N., Saglia, R. P., Goudfrooij, P., Kissler-Patig, M., Maraston, C., Scwheizer, F., \& Zoccali, M. 2006 AEA A, 448, 881

Elson, R. A. W., Fall, M. S., \& Freeman, K. C. 1987, ApJ 323, 54 (EFF)

Gieles, M., Bastian, N., Lamers, H. J. G. L. M., \& Mout, J. N. 2005, A\&\&A, 441, 949

Goodwin, S. P. 1997, MNRAS, 284, 785

Goodwin, S. P. \& Bastian, N. 2006, MNRAS, 373, 752

King, I. 1962, AJ 67, 471

Kroupa, P. 2002, Science, 295, 82

Lada, C. J. \& Lada, E. A. 1991, in The formation and evolution of star clusters, ed. K. Joes, ASP Conf. Ser., 13, 3

Maraston, C., Bastian N., Saglia R. P., Kissler-Patig, M., Schweizer, F., Goudfrooij, P. 2004, $A \mathscr{E} A, 416,467$

Pellerin, A., Meyer, M., Harris, J., \& Calzetti, D. 2007, ApJL, 658, L87

Salpeter, E. E. 1955, ApJ, 121, 161

Smith, L. J. \& Gallagher, J. S. 2001, MNRAS, 326, 1027

van Wijngaarden, L. 1968, J. Engng Maths 2, 225

Whitmore, B. C. 2003, in A Decade of HST Science, ed. M. Livio, K. Noll, \& M. Stiavelli (Cambridge: Cambridge University Press), 153 